\title{
Teletraffic performance Analysis of Multi-band Overlaid WCDMA Systems
}

\author{
Wang, Hua; Iversen, Villy Bæk
}

Published in:

3rd Euro NGI 2007

Link to article, DOI:

10.1109/NGI.2007.371219

Publication date:

2007

Document Version

Publisher's PDF, also known as Version of record

Link back to DTU Orbit

Citation (APA):

Wang, H., \& Iversen, V. B. (2007). Teletraffic performance Analysis of Multi-band Overlaid WCDMA Systems. In 3rd Euro NGI 2007 (pp. 218-225). IEEE. https://doi.org/10.1109/NGI.2007.371219

\section{General rights}

Copyright and moral rights for the publications made accessible in the public portal are retained by the authors and/or other copyright owners and it is a condition of accessing publications that users recognise and abide by the legal requirements associated with these rights.

- Users may download and print one copy of any publication from the public portal for the purpose of private study or research.

- You may not further distribute the material or use it for any profit-making activity or commercial gain

- You may freely distribute the URL identifying the publication in the public portal 


\title{
Teletraffic Performance Analysis of Multi-band Overlaid WCDMA Systems
}

\author{
Hua Wang and Villy B. Iversen \\ Department of Communications, Optics \& Materials \\ Technical University of Denmark, Lyngby, Denmark \\ Email: \{huw, vbi\}@com.dtu.dk
}

\begin{abstract}
Wide-band Code Division Multiple Access (WCDMA) systems are considered to be among the best alternatives for Universal Mobile Telecommunication System (UMTS). In future deployment of WCDMA systems, spectrum overlay among sub-bands with different bandwidth is necessary to support various kinds of services. In this paper, we present a novel approach for evaluating the teletraffic performance of multi-band overlaid WCDMA systems in terms of time, call, and traffic blocking probabilities of each service class. We convert the radio model into a circuit switched network by introducing the concept of virtual channel so that classical teletraffic theory can be applied. A service class is modelled as a BPP (Binomial-Poisson-Pascal) multi-rate traffic stream.
\end{abstract}

\section{INTRODUCTION}

Future mobile communication systems will provide not only speech and low-speed data services, but also high-speed data services such as wireless multimedia applications. To support those features, future mobile communication systems should have the capability of supporting high-speed data transmission at the radio interface up to several megabits per second. The Universal Mobile Telecommunication System (UMTS) has been proposed for the 3rd generation mobile communication systems. It promises to provide a vast range of data services with data rates ranging from $2.4 \mathrm{Kbps}$ to $2 \mathrm{Mbps}$. This is achieved by operating with the Wide-band Code Division Multiple Access (WCDMA) over the air interface.

To accommodate various traffic types (voice, low-speed data, and high-speed data), channels of different bandwidths are supported. For example, the predefined bandwidths of WCDMA and CDMA2000 are 1.25/5/10/20 MHz [2]. Furthermore, with different spreading bandwidths, the service provider has flexibility to deploy any combination of channels for various service types. An important issue in the gradual deployment of wide-band CDMA systems is their backward compatibility with current narrow-band CDMA systems. So when a wide-band CDMA system is deployed in the same area as narrow-band CDMA, systems of different spreading spectrums coexist. On the other hand, since the radio spectrum is a very scarce resource, mutual exclusive allocation of spectrum for different spreading bandwidths is inefficient in a multi-band system. Hence a spectrally overlaid multi-band CDMA system seems to be an appropriate way to integrate systems with different bandwidths. The goal of this paper is to contribute to the development of a deeper understanding of the traffic behavior of multi-band overlaid CDMA systems through the analysis approach of converting a radio model into a conventional circuit switched network with minimum/maximum resource allocation.

This paper is organized as follows. Previous works considering capacity analysis of multi-band overlaid CDMA systems are reviewed in section II. In section III, we derive the general capacity formula for multi-band overlaid CDMA systems and introduce the concept of virtual channel bandwidth and unit channel bandwidth. Section IV presents a transformation from radio model to teletraffic model. Traffic model and convolution algorithm are introduced in section V and VI, respectively. Numerical examples are given in section VII. Conclusions are made in the last section VIII. In appendix I the interference approach is summarized.

\section{Previous Work}

Since the first introduction of the concept "Spectrally Overlaid Multi-band CDMA Systems", calculation of system capacity and blocking probabilities has been an area of interesting research. The first paper considering capacity analysis of spectrally overlaid multi-band CDMA systems was published by Jeong \& Kim [1]. The reverse link capacity of a system with three different spreading bandwidths corresponding to three service classes is calculated in terms of the aggregated data rate in each service class satisfying individual QoS requirements. The performance of different spectrum overlay patterns is evaluated and compared. The results show that the system capacity depends heavily on the spectrum overlay pattern. Specifically, the full assignment is the best overlay pattern from viewpoint of capacity, while mutual exclusion is the second best one. Paper [2] is a generalization of [1]. In [2], a decomposition method for the capacity analysis is introduced so that systems with any number of service classes and any spectrum overlay pattern can be analyzed. The reverse link capacity is estimated in terms of the maximum number of simultaneous users supported in each sub-band. Conditions for achieving the maximum bandwidth utilization are also derived in this paper. Paper [6] continues the work of paper [2] to evaluate the teletraffic performance of multi-band overlaid CDMA systems in terms of the blocking probabilities of each traffic stream. The authors first calculate the border states of multi-band overlaid CDMA systems based on the 
decomposition method proposed by Zhuge \& Li [2]. Then the teletraffic theory of multi-dimensional loss system model is introduced to calculate time, call and traffic congestion of each traffic stream.

The performance analysis of spectrally overlaid multi-band CDMA systems is very challenging, as the mixed interference from the overlapping sub-bands is difficult to formulate. The approaches presented in the above mentioned papers are similar. They base their analysis on the results of interference estimation. Generally speaking, this approach can be decomposed into three steps: 1) calculate the interference density in each sub-band of multi-band overlaid CDMA systems; 2) find the aggregated data rate or the state space of the system while satisfying different QoS requirements; 3) calculate the relevant teletraffic parameters based on the state space. However, the interference estimation approach assumes that the interference power from the overlapping sub-bands is uniformly distributed over sub-band $k$, which is not always the case. In fact, the interference density in the fully overlaid part of sub-band $k$ is higher than that in the partial overlaid part. In this paper, we present a novel approach for the teletraffic performance evaluation of spectrally overlaid multi-band CDMA systems by converting the radio model into classical circuit switched teletraffic model with maximum resource allocation. A very powerful algorithm, the convolution algorithm, is introduced to calculate the related teletraffic parameters in terms of time, call, and traffic congestion.

\section{WCDMA SYSTEM CAPACITY}

We consider a single-cell multi-band overlaid CDMA system with an arbitrary overlay pattern. It is well know that the capacity of a CDMA systems is interference limited. A commonly used measurement for quality of service (QoS) in wireless communications is the bit-energy to interferencedensity ratio $\left(E_{b} / I_{o}\right)$, defined as [4]:

$$
\gamma_{k}=\left(\frac{E_{b}}{I_{o}}\right)_{k}=\frac{S_{k}}{v_{k} R_{k}} \cdot \frac{W_{k}}{I_{k}}
$$

where $\left(E_{b}\right)_{k}=S_{k} /\left(v_{k} R_{k}\right)$ is the bit energy in the received signal, $\left(I_{o}\right)_{k}=I_{k} / W_{k}$ is the received interference density, $S_{k}$ is the received signal power at the base station, $v_{k}$ is the activity factor, $R_{k}$ is the data rate, $W_{k}$ is the spreading bandwidth, $I_{k}$ is the total interference power including the thermal noise power received at the base station, and subscript $k$ denotes parameters in the $k$ th sub-band.

If the total interference power exceeds the thermal noise power above a predetermined threshold, the system will become unstable. WCDMA call admission control is performed on basis of the measured noise rise [4], which is defined as the ratio between the total interference power and the thermal noise power:

$$
\text { Noise rise }=\frac{I_{\text {total }}}{N_{0}}=\frac{I_{\mathrm{own}}+I_{\text {other }}+N_{0}}{N_{0}}
$$

The total interference power $I_{\text {total }}$ comprises of own cell interference power $I_{\text {own }}$, other cell interference power $I_{\text {other }}$, and thermal noise power $N_{0}$. A transformation of (2) yields the following expression:

$$
\text { Noise rise }=\frac{1}{1-\frac{I_{\text {own }}+I_{\text {other }}}{I_{\text {own }}+I_{\text {other }}+N_{0}}}=\frac{1}{1-\eta}
$$

where we define the reverse link load factor $\eta$ as [4]:

$$
\eta=\frac{I_{\text {own }}+I_{\text {other }}}{I_{\text {own }}+I_{\text {other }}+N_{0}}
$$

When $\eta$ is equal to 1 , the corresponding noise rise approaches infinity and the system reaches its pole capacity. A typical value of $\eta$ is $0.8-0.9$. Additionally, other cell interference can be modeled as a fraction of own cell interference by a factor $f[4]$ :

$$
f=\frac{\text { other cell interference }}{\text { own cell interference }}=\frac{I_{\text {other }}}{I_{\text {own }}}
$$

Combining Exp. (1), (4) and (5), the reverse link load factor of sub-band $k$ can be written as:

$$
\eta_{k}=(1+f) \cdot \frac{I_{k, \text { own }} \cdot \gamma_{k} R_{k} v_{k}}{S_{k} W_{k}}
$$

where $I_{k, \text { own }}$ is the received own cell interference power in sub-band $k$. Assuming perfect power control, it is approximately equal to the received signal power generated by users in sub-band $k$ plus the received signal power from other sub-bands overlapping with sub-band $k$ [2], which can be expressed as:

$$
I_{k, \text { own }}=N_{k} S_{k}+\sum_{j \in \alpha} N_{j} S_{j} \frac{W_{k}}{W_{j}}+\sum_{j \in \beta} N_{j} S_{j}
$$

where $N_{k}$ is the number of users in sub-band $k$, and $\alpha, \beta$ are two sets of sub-bands defined as:

$$
\begin{aligned}
& \alpha=\left\{i \mid i \neq k \cup W_{k} \subseteq W_{i}\right\} \\
& \beta=\left\{i \mid i \neq k \cup W_{i} \subseteq W_{k}\right\}
\end{aligned}
$$

The maximum number of supportable users in sub-band $k$ is achieved when there is no interference from the overlapping sub-bands $\left(N_{i}=0, \forall i \neq k\right)$. Then the own cell interference in sub-band $k$ is $I_{k \text {,own }}=N_{k} S_{k}$. Let us introduce $p_{k}$ as: $p_{k}=\gamma_{k} R_{k} v_{k}$, which contains all service specific parameters. Based on Exp. (6), the maximum number of supportable users in sub-band $k$ is:

$$
N_{\max , k}=\frac{\eta_{k}}{1+f} \cdot \frac{W_{k}}{p_{k}}
$$

In CDMA systems, users transmit signals inside the entire allocated spectrum simultaneously. The capacity of CDMA is determined through signal and noise power levels and their ratio. Such modeling is very close to the physical characteristics of CDMA systems. However, for teletraffic calculations, it is preferable to convert all radio transmission issues and power levels into channels so that the classical teletraffic theory can be applied to evaluate system performance parameters such as blocking probabilities. For that reason, we need to express different service classes in multi-band overlaid CDMA 


\begin{tabular}{|c|c|c|c|c|c|c|c|c|}
\hline$k$ & Service & $W_{k}[\mathrm{MHz}]$ & $R_{k}[\mathrm{Kbps}]$ & $v_{k}$ & $\gamma_{k}[\mathrm{~dB}]$ & $\eta_{k}$ & $N_{\max , k}$ & $C_{k}[\mathrm{KHz}]$ \\
\hline 1 & Data & 15 & 300 & 1 & 6 & 0.9 & 11.3 & 1327.0 \\
2 & Data & 10 & 100 & 1 & 6 & 0.9 & 22.6 & 442.3 \\
3 & Voice & 5 & 50 & 0.67 & 7 & 0.9 & 26.8 & 186.6 \\
\hline
\end{tabular}

TABLE I

AN EXAMPLE OF CALCULATING THE VIRTUAL CHANNEL BANDWIDTH IN EACH SERVICE CLASS

systems by different bandwidths virtually needed for one connection of a particular service class. We know that $N_{\max , k}$ is the maximum number of supportable users in service class $k$ with a spreading bandwidth of $W_{k}$. Therefore, we define the virtual channel bandwidth of service class $k$ as

$$
C_{k}=\frac{W_{k}}{N_{\max , k}}=(1+f) \cdot \frac{p_{k}}{\eta_{k}}
$$

which is the virtual bandwidth needed to establish one connection of service class $k$. An example of calculating the virtual channel bandwidth in multi-service multi-band CDMA systems is shown in Table I. Other cell interference is not considered.

In multi-service multi-band CDMA systems, different service classes require different virtual channel bandwidths. Thus it would be beneficial for the teletraffic calculation if we could specify a common channel bandwidth which we may call it a unit channel. The higher the required accuracy (bandwidth granularity), the smaller the unit channel we have to specify [5]. An example of choosing the unit channel bandwidth in multi-service multi-band CDMA systems based on Table I is shown in Table II, where $C_{\text {unit }}$ is the unit channel bandwidth, $d_{k}$ is number of unit channels needed to establish one connection of service class $k$, and $n_{k}$ is total number of unit channels in sub-band $k$.

\begin{tabular}{|c|c|c|c|c|c|c|}
\hline$k$ & Service & $W_{k}[\mathrm{MHz}]$ & $C_{k}[\mathrm{KHz}]$ & $C_{\text {unit }}[\mathrm{KHz}]$ & $d_{k}$ & $n_{k}$ \\
\hline 1 & Data & 15 & 1327.0 & 10 & 133 & 1500 \\
2 & Data & 10 & 442.3 & 10 & 44 & 1000 \\
3 & Voice & 5 & 186.6 & 10 & 19 & 500 \\
\hline
\end{tabular}

TABLE II

Number OF VIRTUAL CHANNELS NEEDED IN EACH SERVICE CLASS

As an approximation, we may choose a unit channel bandwidth equal to $10 \mathrm{KHz}$. Then the three service classes shown in Table I require 133, 44, and 19 unit channels to establish one connection respectively.

\section{Classical Teletraffic Model for Multi-Band OVERLAID CDMA SYSTEMS}

In the previous section, we have converted the transmission issues into virtual channels so that traffic analysis can be separated from transmission issues. Now we can evaluate the teletraffic performance of multi-service multi-band overlaid CDMA systems by using the classical teletraffic model of multi-dimensional loss systems with maximum resource allocation.
In service integrated systems (B-ISDN and UMTS), several service classes are offered to a single system. Without loss of generality, we assume that every sub-band in multi-band overlaid CDMA systems contains one service class only. For sub-bands which contain multiple service classes, they can be separated into identical fully overlaid sub-bands [2].

Let us consider a multi-service single-band CDMA system first. In such a system where all users belonging to different service classes transmit over the entire allocated system bandwidth, users have full accessibility to all channels, but at any instant the following restriction to the number of simultaneous calls in service class $k$ has to be satisfied:

$$
\sum_{k=1}^{N} d_{k} i_{k} \leq n
$$

where $i_{k}$ is the actual number of users in service class $k$, $d_{k}$ is the number of virtual channels needed to establish a connection of service class $k$, and $n$ is the total number of system virtual channels calculated as the system bandwidth divided by the unit channel bandwidth.

The above restriction is equivalent to the restrictions of a circuit switched communication network. In general, for an arbitrary overlay pattern of multi-service multi-band overlaid CDMA systems, there could be several restrictions to the number of simultaneous calls that each service class can support. The basic idea is to set the restrictions at each sub-band, and transform the radio model into an equivalent circuit switched network with minimum/maximum resource allocation as shown in the following.

A circuit switched network model is described by number of traffic streams $K$, number of links $L$, and number of virtual channels $d_{k}$ needed to establish a connection of stream $k$. A link corresponds to a restriction and has a limited capacity $n_{i}$, which is the maximum accessible number of virtual channels associated with link $i$. A traffic stream corresponds to a service class. The number of links is equal to the number of restrictions. The sum of virtual channels used by all traffic streams on link $i$ is thus limited by $n_{i}$.

In multi-band overlaid CDMA systems, sub-bands with different spreading bandwidths are spectrally overlaid. Unlike single-band CDMA systems where all users have full access to all channels, users in different sub-bands of multi-band overlaid CDMA systems have different access to channels, and the accessability depends on the spectrum overlay pattern. Further restrictions have to be introduced in modeling such overlaid systems by using classical teletraffic models.

For two overlapping sub-bands $i$ and $j$, assuming $W_{i} \subset$ 
$W_{j}$, the number of virtual channels occupied in the narrower sub-band $i$ from the broader sub-band $j$ is a fraction $W_{i} / W_{j}$ of the number of occupied virtual channels in sub-band $j$. On the other hand, the number of virtual channels occupied in the broader sub-band $j$ from the narrower sub-band $i$ is equal to the number of occupied virtual channels in sub-band $i$. Therefore, the total number of occupied virtual channels in sub-band $k$ consists of the virtual channels occupied by own sub-band users and a fraction of virtual channels occupied by the overlapping sub-bands. The general restriction to number of simultaneous calls in service class $k$ becomes:

$$
d_{k} i_{k}+\sum_{m \in \alpha} d_{m} i_{m}+\sum_{n \in \beta} d_{n} i_{n} \frac{W_{k}}{W_{n}} \leq n_{k}
$$

where $n_{k}$ is the total number of virtual channels in sub-band $k$, calculated as the bandwidth of sub-band $k$ divided by the unit channel bandwidth, and $\alpha, \beta$ are two sets of sub-bands of which have been defined previously.

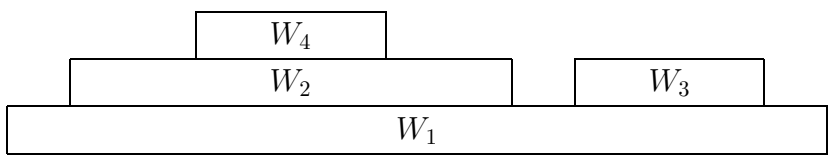

Fig. 1. Example of a multi-band overlaid CDMA system

Consider a multi-band overlaid CDMA system shown in Figure 1 as an example, where each rectangle represents a sub-band sharing the system bandwidth, and the width of the rectangle represents the bandwidth $W_{k}$ occupied by sub-band $k$. We assume that there is always a sub-band spanning the entire system bandwidth ( $W_{1}$ in Fig. 1). Restrictions to the number of simultaneous calls in each service class $k$ are as follows, by (11):

$$
\begin{aligned}
\sum_{k=1}^{4} d_{k} i_{k} & \leq n_{1}, \\
d_{1} i_{1} \frac{W_{2}}{W_{1}}+d_{2} i_{2}+d_{4} i_{4} & \leq n_{2}, \\
d_{1} i_{1} \frac{W_{3}}{W_{1}}+d_{3} i_{3} & \leq n_{3}, \\
d_{1} i_{1} \frac{W_{4}}{W_{1}}+d_{2} i_{2} \frac{W_{4}}{W_{2}}+d_{4} i_{4} & \leq n_{4} .
\end{aligned}
$$

The equivalent circuit switched network is shown in Figure 2, with the route/link matrix table specified in Table III, where the columns in the table indicate the number of virtual channels occupied in each link for establishing a connection of a specific service class.

\section{TRAFFIC MOdEL}

Each service class in a service integrated system corresponds to a traffic stream. Several traffic streams are offered to the multi-band overlaid CDMA systems. Each traffic stream may have individual traffic parameters and may be a statedependent Poisson arrival process with multi-slot traffic. We use the BPP (Binomial \& Poisson \& Pascal) traffic model

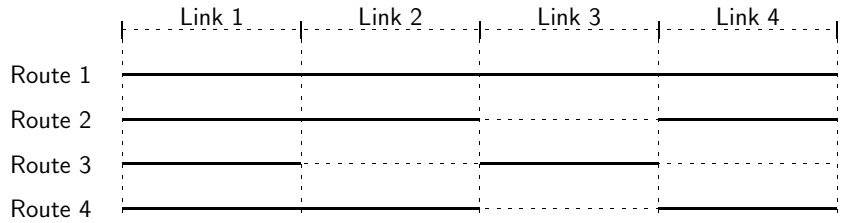

Fig. 2. Classical teletraffic model of a multi-service multi-band overlaid CDMA system shown in Fig. 1.

\begin{tabular}{|c|c|c|c|c|c|}
\hline Link & Class 1 & Class 2 & Class 3 & Class 4 & Capacity \\
\hline 1 & $d_{1}$ & $d_{2}$ & $d_{3}$ & $d_{4}$ & $n_{1}$ \\
2 & $d_{1} \frac{W_{2}}{W_{1}}$ & $d_{2}$ & 0 & $d_{4}$ & $n_{2}$ \\
3 & $d_{1} \frac{W_{3}}{W_{1}}$ & 0 & $d_{3}$ & 0 & $n_{3}$ \\
4 & $d_{1} \frac{W_{4}}{W_{1}}$ & $d_{2} \frac{W_{4}}{W_{2}}$ & 0 & $d_{4}$ & $n_{4}$ \\
\hline
\end{tabular}

TABLE III

LINK-ROUTE TABLE FOR AN EQUIVALENT CIRCUIT SWITCHED NETWORK OF A MULTI-SERVICE MULTI-BAND CDMA SYSTEM SHOWN IN FIG. 1.

in our analysis. This model is insensitive to the service time distributions. Each traffic stream may have its own holding time distribution. Thus it is very robust for applications. The traffic stream $i$ is characterized by the mean offered traffic $A_{i}$, the peakedness $Z_{i}$ and the number of unit channels $d_{i}$ needed for establishing one connection. The offered traffic $A_{i}$ is usually defined as the average number of call attempts per mean holding time. Peakedness $Z_{i}$ is the variance/mean ratio of the state probabilities in a system with infinite capacity and it characterizes the arrival process. For $Z_{i}=1$ we have a Poisson arrival process, whereas $Z_{i}>1$ corresponds to a more bursty Pascal arrival process (Pareto inter-arrival times). For $Z_{i}<1$, we have a finite number of users (Engset case) and more smooth traffic [3].

\section{Vi. Convolution Algorithm for TeletrafFic PERFORMANCE ANALYSIS}

We consider a system consisting of $n$ homogeneous channels. Being homogeneous means that they have the same service rate. The system is offered $N$ different types of traffic streams with maximum resource allocation $m_{k}$ for stream $k$. A call of type $k$ requires $d_{k}$ channels during the whole service time, i.e. all $d_{k}$ channels are occupied and released simultaneously. The arrival processes are general state-dependent Poisson processes. We can model this system as a $N$-dimensional loss system. If it is a reversible Markov process, and it has the product form property, the numerical evaluation can be done by using the convolution algorithm first introduced by Iversen [3]. The condition for reversibility is that Kolmogorov's criteria must be fulfilled for all possible paths [3]. In practice, we experience no problems, because the solution obtained under the assumption of reversibility will be the correct solution if and only if the node balance equations are fulfilled. In the following, we use this as the basis for introducing the convolution algorithm. 
The algorithm consists of three steps:

- Step 1: Calculate the normalized state probabilities of each traffic steam in the corresponding sub-band as if it was alone in the system:

$$
P_{k}=p_{k}(0), p_{k}(1), \ldots, p_{k}\left(m_{k}\right) \quad k=1,2, \ldots, N
$$

- Step 2: Calculate the aggregated state probabilities of all traffic steams except the $i$ th traffic stream by successive convolutions:

$$
Q_{N / i}=P_{1} * P_{2} * \ldots * P_{i-1} * P_{i+1} \ldots * P_{N}
$$

The convolution operator $*$ is defined as

$$
\begin{gathered}
P_{i} * P_{j}=\left\{p_{i}(0) \cdot p_{j}(0), \sum_{x=0}^{1} p_{i}(x) \cdot p_{j}(1-x), \ldots\right. \\
\left.\ldots, \sum_{x=0}^{u} p_{i}(x) \cdot p_{j}(u-x)\right\}
\end{gathered}
$$

where

$$
u=\min \left\{n_{i}+n_{j}, n\right\}
$$

- Step 3: By convolution with the last stream $i$, we get the aggregated state probabilities for the total systems:

$$
P(j)=\frac{Q_{N}(j)}{Q}=\frac{1}{Q} \sum_{x=0}^{j} Q_{N / i}(j-x) \cdot p_{i}(x)
$$

where $Q$ is the normalization constant:

$$
Q=\sum_{j=0}^{n} Q_{N}(j)
$$

The time congestion, call congestion and the traffic congestion can be calculated during the last convolution with some of the intermediate results stored through the convolutions. Steps 2-3 are repeated for every traffic stream.

For a network with more links the procedure is the same, but the truncation of the state space is more complex. More details are given in the Teletraffic Engineering Handbook [3].

\section{NUMERICAL EXAMPLES}

We now consider a multi-band overlaid CDMA system with two basic overlay patterns described in Figure 3. It consists of three different spreading bandwidths corresponding to three service classes. The system parameters of each sub-band are listed in Table I. Three traffic streams listed in Table IV are offered to the system.

\begin{tabular}{|c|c|c|c|c|}
\hline $\begin{array}{c}\text { Service } \\
k\end{array}$ & $\begin{array}{c}\text { Offered traffic } \\
A_{k} \text { [erlang] }\end{array}$ & $\begin{array}{c}\text { Peakedness } \\
Z_{k}\end{array}$ & $\begin{array}{c}\text { Channels } \\
n_{k}\end{array}$ & $\begin{array}{c}\text { Slot needed } \\
d_{k}\end{array}$ \\
\hline 1 & 2 & 2 & 1500 & 133 \\
2 & 14 & 0.5 & 1000 & 44 \\
3 & 16 & 1 & 500 & 19 \\
\hline
\end{tabular}

TABLE IV

TRAFFIC PARAMETERS FOR THE CASE CONSIDERED

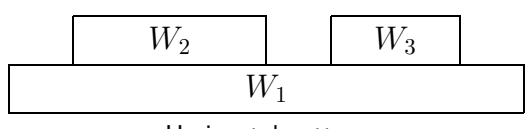

Horizontal pattern

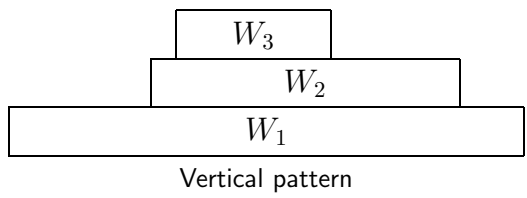

Fig. 3. Example of multi-band overlaid CDMA systems with two overlay patterns

Sub-band 1 has a bandwidth of $15 \mathrm{MHz}$ and the data rate is $300 \mathrm{Kbps}$. It is called "wide-band CDMA (W-CDMA)" subsystem. Traffic stream 1 offered to sub-band 1 has an offered traffic of 2 erlang and peakedness of 2 . It can be classified as Pascal traffic, which means that it is a bursty traffic. Thus the traffic carried in sub-band 1 can be high-speed data traffic.

Sub-band 2 has a bandwidth of $10 \mathrm{MHz}$ and the data rate is $100 \mathrm{Kbps}$. It is called "medium-band CDMA (M-CDMA)" subsystem. Traffic stream 2 offered to sub-band 2 has an offered traffic of 14 erlang and peakedness of 0.5 . It can be classified as Engset traffic, which means that it is a smooth traffic. Thus the traffic carried in sub-band 2 can be low-speed streaming traffic from a finite number of 28 users.

Sub-band 3 has a bandwidth of $5 \mathrm{MHz}$ and the data rate is $50 \mathrm{Kbps}$. It is called "narrow-band CDMA (N-CDMA)" subsystem. Traffic stream 3 offered to sub-band 3 has an offered traffic of 16 erlang and peakedness of 1 . It can be classified as Poisson traffic, which means that it is a random traffic. Thus the traffic carried in sub-band 3 can be voice traffic.

We assume perfect power control, and other cell interference is not considered although it can easily be included. Using the approach introduced in section IV, we get the corresponding teletraffic models of multi-band overlaid CDMA systems as discussed in the following.

\section{A. Horizontal Pattern}

The corresponding teletraffic model for the multi-band overlaid CDMA system with horizontal overlay pattern described in Figure 3 is shown in Figure 4 with restrictions of:

$$
\begin{array}{r}
\sum_{k=1}^{3} d_{k} i_{k} \leq n_{1}, \\
d_{1} i_{1} \frac{W_{2}}{W_{1}}+d_{2} i_{2} \leq n_{2}, \\
d_{1} i_{1} \frac{W_{3}}{W_{1}}+d_{3} i_{3} \leq n_{3} .
\end{array}
$$

The above restrictions are equivalent to the route/link matrix shown in Table V.

The blocking probabilities of each traffic stream in horizontal overlay pattern are shown in Table VI. By using the 


\begin{tabular}{|c|c|c|c|c|c|c|r|r|}
\hline Class & \multicolumn{2}{|c|}{ Time Congestion } & \multicolumn{2}{c|}{ Traffic Congestion } & \multicolumn{2}{c|}{ Call Congestion } & \multicolumn{2}{c|}{ Carried Traffic } \\
\hline & teletraf & interfer & teletraf & interfer & teletraf & interfer & teletraf & interfer \\
\hline 1 & 0.1683 & 0.1647 & 0.3743 & 0.3716 & 0.2302 & 0.2282 & 1.2514 & 1.2568 \\
2 & 0.0426 & 0.0427 & 0.0180 & 0.0180 & 0.0354 & 0.0354 & 13.7477 & 13.7477 \\
3 & 0.0329 & 0.0300 & 0.0329 & 0.0300 & 0.0329 & 0.0300 & 15.4730 & 15.5196 \\
\hline
\end{tabular}

TABLE VI

HORIZONTAL PATTERN. COMPARISON OF BLOCKING PROBABILITIES OF EACH TRAFFIC STREAM OBTAINED BY USING THE TELETRAFFIC APPROACH AND THE INTERFERENCE ESTIMATION APPROACH RESPECTIVELY.

\begin{tabular}{|c|c|c|c|c|}
\hline Link & Class 1 & Class 2 & Class 3 & Capacity \\
\hline 1 & $d_{1}$ & $d_{2}$ & $d_{3}$ & $n_{1}$ \\
2 & $d_{1} \frac{W_{2}}{W_{1}}$ & $d_{2}$ & 0 & $n_{2}$ \\
3 & $d_{1} \frac{W_{3}}{W_{1}}$ & 0 & $d_{3}$ & $n_{3}$ \\
\hline
\end{tabular}

TABLE V

EQUIVALENT CIRCUIT SWITCHED NETWORK OF MULTI-SERVICE MULTI-BAND CDMA SYSTEMS WITH HORIZONTAL PATTERN.

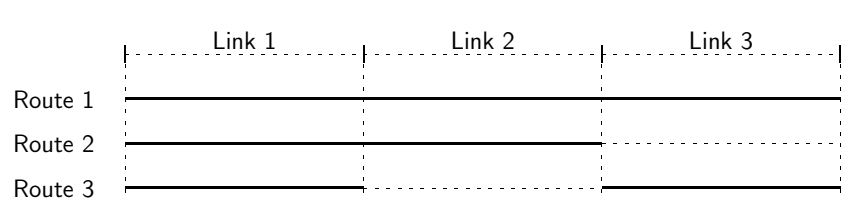

Fig. 4. Classical teletraffic model for multi-band overlaid CDMA system with horizontal pattern shown in Fig. 3.

convolution algorithm described above, we get the results under heading 'teletraf'. To verify the accuracy of our approach, we calculate the blocking probabilities of each traffic stream by using the interference estimation approach (see Appendix) under heading 'interfer' and compare them with our calculation results.

From the results we see that the results obtained by two different approaches are almost the same.

\section{B. Vertical Pattern}

The corresponding teletraffic model for the multi-band overlaid CDMA system with vertical overlay pattern described in Figure 3 is shown in Figure 5 with restrictions of:

$$
\begin{aligned}
\sum_{k=1}^{3} d_{k} i_{k} & \leq n_{1}, \\
d_{1} i_{1} \frac{W_{2}}{W_{1}}+d_{2} i_{2}+d_{3} i_{3} & \leq n_{2}, \\
d_{1} i_{1} \frac{W_{3}}{W_{1}}+d_{2} i_{2} \frac{W_{3}}{W_{2}}+d_{3} i_{3} & \leq n_{3} .
\end{aligned}
$$

The above restrictions are equivalent to the route/link matrix shown in Table VII.

The blocking probabilities of each traffic stream are obtained by using the convolution algorithm, as shown in Table VIII. We can see that the result obtained by our new teletraffic approach again is in accordance with the interference estimation approach.

\begin{tabular}{|c|c|c|c|c|}
\hline Link & Class 1 & Class 2 & Class 3 & Capacity \\
\hline 1 & $d_{1}$ & $d_{2}$ & $d_{3}$ & $n_{1}$ \\
2 & $d_{1} \frac{W_{2}}{W_{1}}$ & $d_{2}$ & $d_{3}$ & $n_{2}$ \\
3 & $d_{1} \frac{W_{3}}{W_{1}}$ & $d_{2} \frac{W_{3}}{W_{2}}$ & $d_{3}$ & $n_{3}$ \\
\hline
\end{tabular}

TABLE VII

EQUIVALENT CIRCUIT SWITCHED NETWORK OF MULTI-SERVICE MULTI-BAND CDMA SYSTEMS WITH VERTICAL PATTERN.

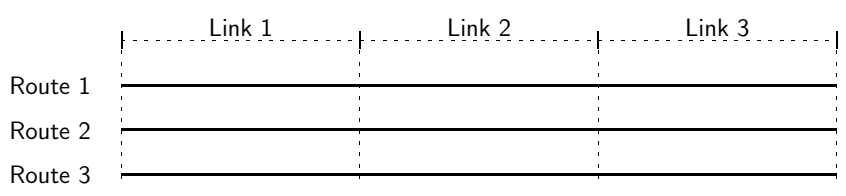

Fig. 5. Classical teletraffic model for multi-band overlaid CDMA system with vertical pattern shown in Fig. 3.

\section{CONCLUSIONS}

In this paper, we have presented a new and innovative approach to evaluate the teletraffic performance of multi-band overlaid CDMA systems in terms of blocking probabilities. The basic idea of the proposed approach is to convert the general radio model of multi-service, multi-band overlaid CDMA systems into a classical circuit switched network with maximum resource allocation by introducing the concept of unit channel bandwidth and virtual channel. Then by applying the convolution algorithm, we are able to evaluate the performance of the system in terms of the time congestion, call congestion and traffic congestion of each traffic stream. By doing so, many existing teletraffic calculation tools can be reused and the computation complexity can be greatly reduced. Our analysis approach introduced in this paper may serve as a good approximation of the teletraffic performance evaluation of multi-band overlaid CDMA systems with an arbitrary overlay pattern, as it is verified by comparing the results of our approach with the traditional interference estimation approach.

\section{REFERENCES}

[1] D.G. Jeong, G. Kim and D. Kim: Capacity analysis of spectrally overlaid multiband CDMA mobile networks, IEEE Transactions on Vehicular Technology, Vol. 47, No. 3, August 1998, pp. 798807.

[2] L. Zhuge and V.O.K. Li: Reverse-link capacity of multiband overlaid DS-CDMA systems, Mobile Networks and Applications, Vol. 7, 2002, pp. 101-113. 


\begin{tabular}{|c|c|c|c|c|c|c|r|r|}
\hline Class & \multicolumn{2}{|c|}{ Time Congestion } & \multicolumn{2}{c|}{ Traffic Congestion } & \multicolumn{2}{c|}{ Call Congestion } & \multicolumn{2}{c|}{ Carried Traffic } \\
\hline & teletraf & interfer & teletraf & interfer & teletraf & interfer & teletraf & interfer \\
\hline 1 & 0.6354 & 0.6262 & 0.7885 & 0.7820 & 0.6509 & 0.6421 & 0.4229 & 0.4359 \\
2 & 0.3790 & 0.3725 & 0.2239 & 0.2196 & 0.3659 & 0.3601 & 10.8655 & 10.9254 \\
3 & 0.3360 & 0.3347 & 0.3360 & 0.3347 & 0.3360 & 0.3347 & 10.6239 & 10.6450 \\
\hline
\end{tabular}

TABLE VIII

VERTICAL PATTERN. COMPARISON OF BLOCKING PROBABILITIES OF EACH TRAFFIC STREAM OBTAINED BY USING THE TELETRAFFIC APPROACH AND THE INTERFERENCE ESTIMATION APPROACH RESPECTIVELY.

[3] V.B. Iversen: Teletraffic Engineering Handbook, COM department, Technical University of Denmark. 2004. 336 pp.

[4] H. Holma and A. Toskala: WCDMA for UMTS: Radio access for third generation mobile communications, 2nd Edition, Wiley, July 2002, ISBN 0-470-84467-1. $391 \mathrm{pp}$.

[5] V.B. Iversen, V. Benetis, N.T. Ha and S. Stepanov: Evaluation of multi-service CDMA networks with soft blocking, 16th ITC Specialist Seminar, Antwerp, Belgium, 2004.

[6] E. Epifania and V.B. Iversen: Teletraffic engineering of multiband W-CDMA Systems, Net-Con 2003, Muscat, Oman, October 2003. Published in Network Control and Engineering for QoS, Security and Mobility II, pp. 90-103, Kluwer Academic Publishers, 2003. 273pp.

\section{APPENDIX I}

\section{INTERFERENCE APPROACH}

The interference estimation approach has been well investigated in [2]. The interference power $I_{k}$ in sub-band $k$ is approximately equal to the received signal power generated by own sub-band users and a fraction of the signal power generated by other sub-bands overlapping with sub-band $k$ plus the thermal noise power, expressed as [2]:

$$
I_{k}=N_{k} S_{k}+\sum_{i \in \alpha} N_{i} S_{i} \frac{W_{k}}{W_{i}}+\sum_{i \in \beta} N_{i} S_{i}+n_{o} W_{k}
$$

where $n_{o}$ is the thermal noise power density. Combined with Exp. (1), the interference density in each sub-band can be written as:

$$
I_{k, o}=\frac{N_{k}}{\phi_{k}} I_{k, o}+\sum_{i \in \alpha} \frac{N_{i}}{\phi_{i}} I_{i, o}+\sum_{i \in \beta} \frac{N_{i}}{\phi_{i}} I_{i, o} \frac{W_{i}}{W_{k}}+n_{o}
$$

where $I_{k, o}=I_{k} / W_{k}$ is the interference density in sub-band $k$, $\Phi_{k}=W_{k} /\left(R_{k} \gamma_{k} v_{k}\right)$ is the maximum number of supportable users in sub-band $k$ when there is no interference from the overlapping sub-bands.

The condition limiting the capacity of multi-band overlaid CDMA systems is that the noise rise should not exceed a predefined threshold in every sub-band, expressed as:

$$
\frac{I_{k, o}}{n_{o}}<\frac{1}{1-\eta_{k}}
$$

where $\eta_{k}$ is the reverse link load factor in sub-band $k$.

In multi-band overlaid CDMA systems, the interference density in each sub-band is not uniformly distributed due to the partial overlay pattern. Furthermore, Exp. (13) implies that the very "top" sub-band always has the highest interference density. Thus we only have to guarantee $I_{t o p, o}<n_{o} /\left(1-\eta_{k}\right)$, and the noise rise constraint is satisfied in all other sub-bands. Taking Fig. 1 as an example, it is straightforward to see that the condition limiting the system capacity is the interference density of sub-band 4 and 3 .

Based on this observation, we can derive the capacity of multi-band overlaid CDMA systems in terms of the number of simultaneous users supported in each sub-band. The system we considered is shown in Fig. 3.

\section{A. Vertical pattern}

In the vertical pattern, we only have to guarantee $I_{3, o}<$ $n_{o} /\left(1-\eta_{k}\right)$, then the power constraint is satisfied in all other sub-bands.

The interference power received in sub-band 3 is:

$$
I_{3}=N_{1} S_{1} \frac{W_{3}}{W_{1}}+N_{2} S_{2} \frac{W_{3}}{W_{2}}+N_{3} S_{3}+n_{o} W_{3}
$$

The corresponding interference density of sub-band 3 is:

$$
I_{3, o}=\frac{N_{1}}{\Phi_{1}} I_{3, o}+\frac{N_{2}}{\Phi_{2}} I_{3, o}+\frac{N_{3}}{\Phi_{3}} I_{3, o}+n_{o}
$$

Thus the capacity boundary condition of the vertical pattern is determined by:

$$
\frac{N_{1}}{\Phi_{1}}+\frac{N_{2}}{\Phi_{2}}+\frac{N_{3}}{\Phi_{3}} \leq \eta
$$

\section{B. Horizontal pattern}

The condition limiting the capacity in the horizontal pattern is that $\max \left(I_{2, o}, I_{3, o}\right)<n_{o} /(1-\eta)$.

The interference power received in sub-band 2 and sub-band 3 are:

$$
\begin{aligned}
& I_{2}=N_{1} S_{1} \frac{W_{2}}{W_{1}}+N_{2} S_{2}+n_{o} W_{2}, \\
& I_{3}=N_{1} S_{1} \frac{W_{3}}{W_{1}}+N_{3} S_{3}+n_{o} W_{3} .
\end{aligned}
$$

The corresponding interference density of sub-band 2 and subband 3 are:

$$
\begin{aligned}
& I_{2, o}=\frac{N_{1}}{\Phi_{1}} I_{2, o}+\frac{N_{2}}{\Phi_{2}} I_{2, o}+n_{o}, \\
& I_{3, o}=\frac{N_{1}}{\Phi_{1}} I_{3, o}+\frac{N_{3}}{\Phi_{3}} I_{3, o}+n_{o} .
\end{aligned}
$$

Thus the capacity boundary condition of the horizontal pattern is determined by:

$$
\max \left(\frac{N_{1}}{\Phi_{1}}+\frac{N_{2}}{\Phi_{2}}, \frac{N_{1}}{\Phi_{1}}+\frac{N_{3}}{\Phi_{3}}\right) \leq \eta
$$




\begin{tabular}{|c|c|c|c|c|c|c|c|c|c|c|c|c|c|c|c|c|c|c|c|c|c|}
\hline sub-band & \multicolumn{21}{|c|}{ border states } \\
\hline 3 & 26 & 25 & 24 & 23 & 22 & 20 & 19 & 18 & 17 & 16 & 14 & $\ldots$ & 5 & 4 & 3 & 1 & 0 & 3 & 1 & 0 & 0 \\
\hline 2 & 0 & 1 & 2 & 3 & 4 & 5 & 6 & 7 & 8 & 9 & 10 & $\ldots$ & 0 & 1 & 2 & 3 & 4 & 0 & 1 & 2 & 0 \\
\hline 1 & 0 & 0 & 0 & 0 & 0 & 0 & 0 & 0 & 0 & 0 & 0 & $\ldots$ & 9 & 9 & 9 & 9 & 9 & 10 & 10 & 10 & 11 \\
\hline
\end{tabular}

TABLE IX

PART OF THE BORDER STATES OF EACH TRAFFIC CLASS IN THE VERTICAL OVERLAY PATTERN.

\begin{tabular}{|c|c|c|c|c|c|c|c|c|c|c|c|c|c|c|c|c|c|c|c|c|c|}
\hline sub-band & \multicolumn{11}{|c|}{ border states } \\
\hline 3 & 26 & 26 & 26 & 26 & 26 & 26 & 26 & 26 & 26 & 26 & 26 & $\ldots$ & 5 & 5 & 5 & 5 & 5 & 3 & 3 & 3 & 0 \\
2 & 0 & 1 & 2 & 3 & 4 & 5 & 6 & 7 & 8 & 9 & 10 & $\ldots$ & 0 & 1 & 2 & 3 & 4 & 0 & 1 & 2 & 0 \\
1 & 0 & 0 & 0 & 0 & 0 & 0 & 0 & 0 & 0 & 0 & 0 & $\cdots$ & 9 & 9 & 9 & 9 & 9 & 10 & 10 & 10 & 11 \\
\hline
\end{tabular}

TABLE $X$

PART OF THE BORDER STATES OF EACH TRAFFIC CLASS IN THE HORIZONTAL OVERLAY PATTERN.

\section{Calculation of blocking probabilities}

Based on expressions (15) and (16), we can calculate the state space of the system. Figure $6 \& 7$ show the border states in terms of the number of supportable users in each sub-band in the vertical and horizontal overlay pattern respectively. Part of the border states are listed in Table IX \& X.

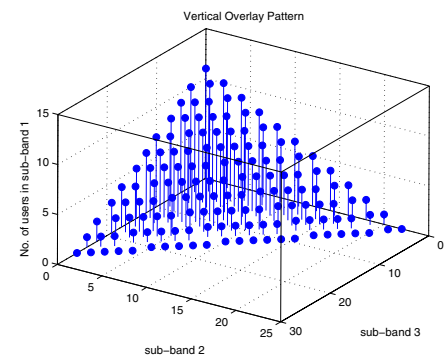

Fig. 6. Number of supportable users in each sub-band in the vertical overlay pattern

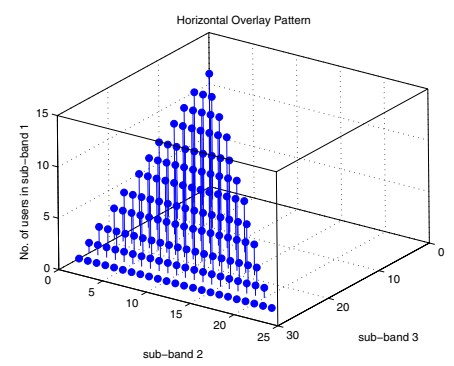

Fig. 7. Number of supportable users in each sub-band in the horizontal overlay pattern

After we get the state space of the system, the teletraffic performance in terms of time, call and traffic congestion can be evaluated by applying the classical multi-dimensional loss system models as shown in Figure 8.

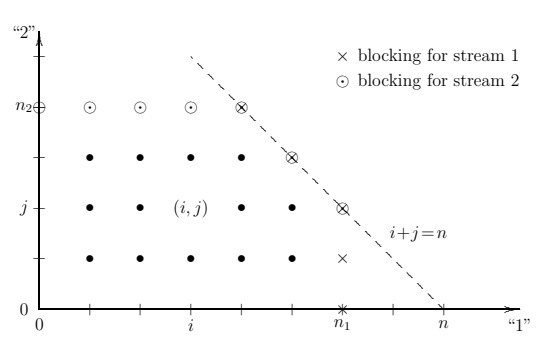

Fig. 8. Structure of the state transition diagram for two-dimensional traffic processes with class limitations. When calculating the equilibrium probabilities, state $(i, j)$ can be expressed by state $(i, j-1)$ and recursively by state $(i, 0),(i-1,0)$, and finally by $(0,0)$. [3] 